Fecha de recepción: 30 de agosto de 2018.

Fecha de evaluación: 18 de junio de 2019. Fecha de aprobación: $1^{\circ}$ de julio de 2020.

\section{Comunidades indígenas del Cauca y la participación
política: 1991, 1992, 1994 y $1998^{*}$}

\section{María Andrea Palta Limas*}

Para citar este artículo

Palta, M. (2020). Comunidades indígenas del Cauca y la participación política: 1991, 1992, 1994 y 1998 Vía luris, 29, 111-127. DOI: https:// doi.org/10.37511/viaiuris.n29a4

\title{
RESUMEN
}

El presente estudio centra su atención en las comunidades indígenas del departamento del Cauca y los procesos de lucha y organización que han llevado a cabo y han permitido su presencia en escenarios de participación política, negociación y procesos de vinculación para la toma decisiones de las poblaciones indígenas en la nación. Para ello se tiene como objetivo analizar y contrastar la evolución de la participación política de estas comunidades en el proceso electoral que se llevó a cabo desde la creación de la Constitución de 1991 hasta el año 1998, enfatizando en las elecciones de la Asamblea Constituyente, Senado, Cámara de Representantes, gobernación y alcaldías, las cuales serán un visor para referenciar el progreso de la participación. Este artículo se estructura haciendo uso de métodos cuantitativos y cualitativos, en un primer momento por medio de la revisión de datos estadísticos como un elemento fundamental para el diagnóstico, toma de decisiones de los actores políticos en este caso, en el que es necesaria la construcción de unidades de análisis y variables que nos permitan la medición de la participación política en los procesos electorales; esta metodología, en complemento con el análisis, documental logra establecer indicadores que dan cuenta de la población que acude a las urnas, niveles de educación de los candidatos; aumentos y disminuciones de las curules conseguidas.

* $\quad$ Este artículo es resultado del proyecto de investigación titulado: Participación política de las comunidades indígenas del departamento del Cauca entre 1991, 1992, 1994 y 1998. Grupo de investigación actores procesos e instituciones políticas. Universidad de Cauca. (Período 2017-2018). Popayán (Colombia).

** Estudiante del programa de Ciencia Política de la Universidad del Cauca, Integrante del semillero de investigación LVMEN adscrito al programa de Ciencia Política (Popayán, Colombia). Correo electrónico de contacto: bandreapalta@unicauca.edu.co. ORCID: https://orcid. org/0000-0002-9001-5913.
DOI: https://doi.org/10.37511/viaiuris.n29a4

Licencia Creative Commons

Esta obra está bajo una Licencia Creative Commons Atribución-NoComercial-Compartirlgual 2.5 Colombia.

\section{cC)(1)(2)}

\section{Palabras clave}

Participación política; comunidades indígenas; elecciones; Constitución política; Cauca. 


\title{
The indigenous communities of Cauca and political participation: 1991, 1992, 1994 and 1998
}

\section{María Andrea Palta Limas}

\begin{abstract}
This study focuses its attention on the indigenous communities of the department of Cauca and the processes of struggle and organization they have carried out and have allowed their presence in scenarios of political participation, negotiation and processes of linkage for decision making of the indigenous populations in the nation. The objective is to analyze and contrast the evolution of the political participation of these communities in the electoral process that took place from the creation of the 1991 Constitution until 1998, emphasizing the elections of the Constituent Assembly, Senate, House of Representatives, governorship and mayoralties, which will be a viewpoint to reference the progress of participation. This article is structured making use of quantitative and qualitative methods, at first through the review of statistical data as a fundamental element for the diagnosis and decision making of the political actors in this case, in which it is necessary the construction of analysis units and variables that allow us to measure the political participation in the electoral processes; this methodology, in complement with the documentary analysis, establishes indicators that show the population that goes to the polls, education levels of the candidates, increases and decreases in the number of seats obtained.
\end{abstract}

\section{Keywords}

Political participation; indigenous communities; elections; political constitution; Cauca; political participation; Cauca. 


\section{As Comunidades Indígenas Do Cauca E A Participação Política: 1991, 1992, 1994 E 1998}

\section{María Andrea Palta Limas}

\section{RESUMO}

Este estudo centra a sua atenção nas comunidades indígenas do departamento de Cauca, nos processos de luta e organização que têm sido levados a cabo permitindo a sua presença em cenários de participação política, negociação e processos de ligação para a tomada de decisões das populações indígenas na nação, O objectivo é analisar e contrastar a evolução da participação política destas comunidades no processo eleitoral que teve lugar desde a criação da Constituição de 1991 até 1998, enfatizando apenas as eleições da Assembleia Constituinte, Senado, Câmara dos Representantes, Gabinete do Governador e Câmaras Municipais, o que será um ponto de vista para referenciar o progresso da participação. Este artigo está estruturado utilizando métodos quantitativos e qualitativos, primeiro através da revisão dos dados estatísticos como elemento fundamental para o diagnóstico e tomada de decisão dos actores políticos neste caso, é necessário construir unidades de análise e variáveis que nos permitam medir a participação política nos processos eleitorais; Em contraste com a análise documental, é possível estabelecer indicadores que mostram a população que vai às urnas, os níveis de educação dos candidatos, o aumento e a diminuição do número de lugares obtidos.

\section{Palavras-chave}

Participação política; comunidades indígenas; eleições; constituição política; Cauca. 


\section{Les Communautés}

indigènes du Cauca et la participation politique : 1991, 1992, 1994 Et 1998

\section{María Andrea Palta Limas}

\section{RÉSUMÉ}

Cette étude concentre son attention sur les communautés indigènes du département de Cauca, les processus de lutte et d'organisation qui ont été réalisés permettant leur présence dans des scénarios de participation politique, de négociation et de processus de liaison pour la prise de décision des populations indigènes dans la nation, L'objectif est d'analyser et de contraster l'évolution de la participation politique de ces communautés dans le processus électoral qui s'est déroulé depuis la création de la Constitution de 1991 jusqu'à 1998, en mettant l'accent uniquement sur les élections de l'Assemblée constituante, du Sénat, de la Chambre des représentants, du bureau du gouverneur et des maires, qui seront un point de vue pour référencer le progrès de la participation. Cet article est structuré en utilisant des méthodes quantitatives et qualitatives, d'abord par le biais de la révision des données statistiques comme élément fondamental pour le diagnostic et la prise de décision des acteurs politiques; dans ce cas, il est nécessaire de construire des unités d'analyse et des variables qui nous permettent de mesurer la participation politique dans les processus électoraux, qui contrairement à l'analyse documentaire parviennent à établir des indicateurs qui montrent la population qui se rend aux urnes, les niveaux d'éducation des candidats, les augmentations et les diminutions du nombre de sièges obtenus.

\section{Mots-clés}

Participation politique; communautés indigènes; élections; constitution politique; Cauca. 


\section{INTRODUCCIÓN}

Las comunidades indígenas desempeñan un papel muy importante en la historia de Colombia pues constituyen un referente histórico respecto a factores identitarios y organizativos es importante considerar que estos grupos están es importante considerar que estos grupos están marcando la diferencia con sus procesos organizativos, por medio de actos de resistencia desde tiempos remotos, muchos de ellos han quedado sin ser registrados, Ilegando al punto de considerar ausentes a los indígenas en los procesos de relevancia en Colombia.

Las comunidades indígenas comienzan a ser excluidas desde la llegada de los europeos al continente latinoamericano. Rojas (2011) menciona que se llevó a cabo todo un proceso de conquista en el que el interés principal de los europeos era la explotación del oro, y desde esta época los nativos empezaron a ser rechazados en los diferentes escenarios de la sociedad; es por ello que el punto de partida de este análisis consiste en retomar las características principales de las culturas indígenas y las luchas que han emprendido para lograr procesos reivindicativos, así como cuáles son sus retos para lograr vincularse de manera importante y trascendental en la política del país.

Con relación a lo anterior, la participación política ha venido cobrando fuerza al interior de las comunidades indígenas, pues se ha convertido en uno de los objetivos principales en sus planes de vida y la forma en que se busca la reivindicación histórica y social; en este contexto, vale tener en cuenta la tesis de Castoriadis (1997) respecto a las "restricciones históricas": aunque no podamos sondear el origen de las sociedades, sí podemos encontrar fragmentos. Esta teoría aplica exactamente para el caso de las comunidades indígena, ya que hay partes de su historia que no se egistran, pero con lo poco que hay sus tradiciones y prácticas vigentes se puede reestructurar y establecer patrones que determinen el curso de acción a tomar.

La participación de las comunidades indígenas en los procesos políticos del escenario nacional se da formalmente, con la creación de la Constitución colombiana de 1991, que deja en claro una mayor inclusión en la toma de decisiones y en escenarios en los que durante quinientos años existieron fronteras invisibles capaces de limitar la diversidad cultural, el pensamiento de sus comunidades y los beneficios civiles a los que solo la gran estirpe tenía acceso.

Con la Constitución Política de 1991 se reconoce la diversidad cultural de Colombia, por ende las comunidades indígenas tienen la oportunidad de participar en los diferentes escenarios políticos y en las dinámicas de la sociedad en general. El presente trabajo tiene como objetivo analizar y contrastar la evolución de la participación política de estas comunidades en el proceso electoral que se llevó a cabo desde la creación de la Constitución de 1991 hasta el año 1998, enfatizando solo en las elecciones de la Asamblea Constituyente, Senado, Cámara de Representantes, gobernaciones y alcaldías.

El artículo se desarrolla en tres apartados: en primer lugar, una caracterización de las comunidades indígenas del departamento del Cauca, teniendo en cuenta las comunidades existentes en este departamento, en función de las prácticas y conductas tradicionales que aportan a su desarrollo en la arena política: en segundo lugar, se identifica la influencia de los contextos en la toma de decisiones, permitiendo develar lo asertivo o no de su configuración; Finalmente, se aborda la incidencia que debe tener la representatividad de las comunidades indígenas en el país.

\section{Candidatos postulados a las elecciones en representación de las comunidades indígenas del departamento del Cauca 1991, 1992, 1994 y 1998}

El departamento del Cauca es pionero en el desarroIlo de procesos de resistencia y organización de las comunidades indígenas, las cuales se han agrupado en la búsqueda de respeto de sus derechos, procesos de reivindicación e inclusión en la arena política, organización que se consolida en el escenario de la Constituyente de 1991. Las comunidades han tenido proyecciones de sus aspiraciones en el escenario electoral y trabajan en lograr la presencia de sus líderes en las esferas políticas.

\section{Comunidades indígenas existentes en el departamento del Cauca 1991, 1992, 1994 y 1998}

Los pueblos indígenas están situados en el continente latinoamericano desde tiempos remotos, de y enfrentaron hace más de quinientos años la llegada 
de los europeos, quienes impulsaron un proceso de colonización y conquista del continente, arrasando a su paso con las culturas, pensamientos, cosmovisiones y creencias, y violando abruptamente todas las formas de vida de los diferentes pueblos indígenas que existían en ese momento histórico. Desde ahí, algunas de esas culturas o pueblos vienen haciendo un proceso de resistencia contra las ofensas y agresiones por parte de los no indígenas.

Es importante mencionar que durante la época de la conquista y los años posteriores a esta, algunas culturas indígenas fueron desapareciendo debido a las masacres y a otros factores, como: la imposición de otra cultura y de la religión católica, que acabó con las tradiciones y cosmovisiones de los pueblos nativos que existían en ese momento.

No obstante como lo menciona el Consejo Regional Indígena del Cauca-CRIC (2007), para la década de los sesenta y los setenta, aún pervivían diferentes pueblos indígenas en el departamento del Cauca, como lo son el pueblo nasa, con más de 110000 habitantes, y conservan su propia lengua Ilamada nasa yuwe; el pueblo misak, con más de 18000 habitantes, conservando su propia lengua Ilamada namuy wan o nam trik; el pueblo yanakona, con alrededor de 24000 habitantes, actualmente están haciendo un proceso de recuperación de la lengua originaria, con procesos de fortalecimiento de la lengua tradicional en comunidades. Ellos fueron quienes inicialmente empezaron a organizarse estratégicamente para defender y proteger sus culturas, es decir, para continuar de alguna u otra manera con el legado de lucha y resistencia que ya se venía trabajando desde la conquista.
Pproceso de resistencia, el 24 de febrero de 1971 se creó el Consejo Regional Indígena del Cauca (CRIC) en el Resguardo de Toribío, con la participación de diferentes pueblos indígenas como misak, nasa, yanakona, kokonuco y totoroez (CRIC, 2007). La preocupación por organizarse empezó por el despojo de las tierras pertenecientes a los campesinos e indígenas por parte de los terratenientes; en este sentido, Duque (2014) menciona que la idea inicial del CRIC era desarrollar un programa de acción orientado la recuperación y ampliación de las tierras pertenecientes a sus resguardos, para de esta manera también fortalecer el poder de las autoridades tradicionales, dar a conocer leyes que los afectaban, iniciar un proceso de reivindicación para defender su historia, lenguas y costumbres e impulsar la implementación de una educación bilingüe y bicultural.

Los procesos de organización se convierten en los puntos de encuentro de los diferentes pueblos indígenas, para que cooperen en el fortalecimiento de su identidad en un trabajo hacia adentro, haciendo una proyección al resto de la sociedad colombiana. Esta organización, fue pionera en todo el movimiento indígena a nivel nacional, ya que fue el inicio de la conformación de muchas otras organizaciones en diferentes departamentos y organizaciones a nivel nacional; también fue un impulso para formar partidos políticos dentro de este movimiento (figura 1).

Para el año 1991, el Consejo Regional Indígena del Cauca (CRIC) que fue creado exclusivamente por las comunidades indígenas de este departamento representa el $66,7 \%$ de la creación de estos movimientos u organizaciones. Por su parte, y así como

Figura 1. Promedio de movimientos u organizaciones indígenas en el Cauca

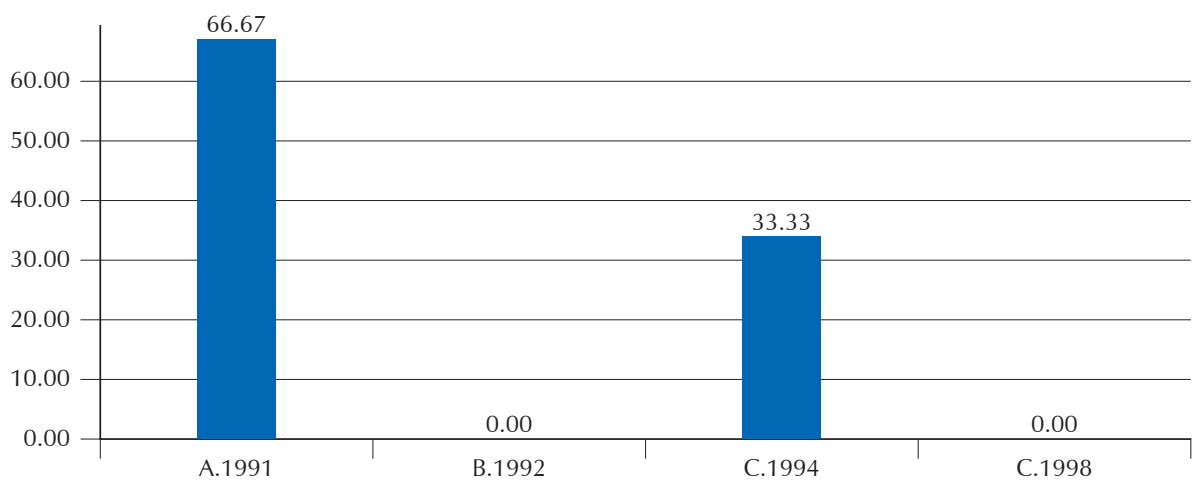

Fuente: Elaboración propia, a partir de información de las organizaciones de los movimientos indígenas en Colombia. 
lo describe Ministerio del Interior (2016), las Autoridades Indígenas de Colombia (AICO), que surgen en el suroccidente colombiano departamentos de Cauca y Nariño son consideradas como una organización indígena y un partido político colombiano; se crearon en el año 1978 con el nombre de Autoridades Indígenas del Sur Occidente Colombiano (AISO), pero desde 1987 hasta la actualidad toman el nombre de AICO.

En 1992 no se registran nuevas organizaciones en el Cauca; en 1994 hubo una representación del 33,3 $\%$, correspondiente a la creación de una organización Ilamada Asociación de Cabildos Indígenas del Norte del Cauca (ACIN); en 1998 tampoco se conformaron otras organizaciones, sin embargo, varias de estas organizaciones continúan vigentes, si bien algunas han cambiado su razón social e intereses políticos.

En este sentido, es necesario resaltar la creación de las organizaciones en diferentes departamentos, ya que estas ayudaron a fortalecer el movimiento indígena tanto en el departamento del Cauca como a nivel nacional. En la figura 2 se representa la creación de organizaciones indígenas regionales en el periodo 1991 (figura 2).

En el año 1991 el número de organizaciones indígenas corresponde al 75 \% las cuales fueron: la Organización Nacional Indígena de Colombia (ONIC), creada en 1982 con la participación de la mayoría de los pueblos indígenas de Colombia para ser representados a nivel nacional de una forma legal como legítima (ONIC, 2016); el Consejo Regional
Indígena del Tolima (CRIT) que surgió en el Tolima también en el mismo año; la Confederación Indígena Tayrona (CIT) que se creó en 1983 en representación del pueblo arhuaco y agrupa los departamentos del Cesar, Guajira y Magdalena.

En el año 1985 surgen dos organizaciones: la Organización Indígena de Antioquia (OIA) y la Organización Regional Embera Waunana (OREWA), que se consolida en el departamento del Chocó. Por su parte, la Organización Zonal Indígena del Putumayo (OZIP) se creó en 1986 en el Putumayo, en tanto que la Alianza Social Independiente (ASI) se empezó a llamar de esta manera desde 1991, aunque inicialmente se llamó Alianza Social Indígena, que además de ser una organización también se constituye como un partido político., Por último, las AICO también hacen parte de las organizaciones regionales porque, como se había mencionado anteriormente esta organización se creó entre el Cauca y Nariño posteriormente se constituyó como un partido político.

En el año 1992 no se tiene registro de conformación de organizaciones, sino que siguieron vigentes las que ya existían; para el año 1994 se obtuvo un 8,3\%, dado que en 1993 se creó el Movimiento Indígena Colombiano (MIC) en representación de los pueblos indígenas a nivel nacional, que se consolida también como un partido político. En 1998 le corresponde un 16,7 \%, ya que en 1995 había surgido la Organización de los Pueblos Indígenas de la Amazonía Colombiana (OPIAC), que agrupa a los pueblos indígenas amazónicos, y en 1998 se

Figura 2. Promedio de organizaciones indígenas regionales

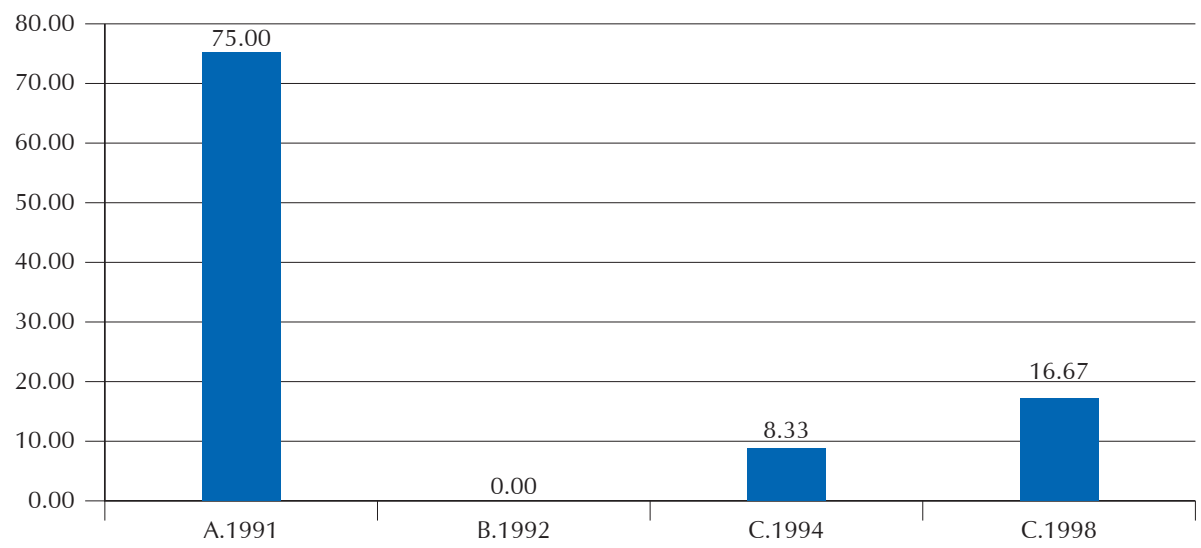

Fuente: Elaboración propia, a partir de información de las organizaciones de los movimientos indígenas en Colombia. 
crea la Unidad Indígena del Pueblo Awá (UNIPA) en Nariño.

Así fue como el movimiento indígena empezó a fortalecer su proceso de lucha y resistencia a nivel local y nacional, desde los años setenta y ochenta, después de la creación del CRIC hasta la actualidad; se ha evidenciado que esas mismas organizaciones se han venido fortaleciendo porque con la Constitución de 1991 han tenido la oportunidad de participar en los procesos electorales.

\section{RESULTADOS Y DISCUSIÓN}

\section{Representantes o candidatos del movimiento indígena en el departamento del Cauca}

Con la constituyente de 1991, los movimientos no se hacen esperar y lanzan sus primeros candidatos indígenas en 1990 para la Asamblea Nacional Constituyente: Lorenzo Muelas Hurtador, indígena misak del municipio de Guambía, elegido por las Autoridades Indígenas de Colombia (AICO), Francisco Rojas Birry, indígena del departamento del Chocó elegido por la Organización Nacional Indígena de Colombia (ONIC) y Alfonso Peña Chepe, indígena nasa del municipio de Caldono y desmovilizado del movimiento armado Quintín Lame. Laurent (2005) indica que la solicitud de estos candidatos era un país más incluyente, tolerante y justo, por lo cual pedían que se tuvieran en cuenta sus derechos y la garantía de estos en la nueva Constitución Política.

En este mismo año se realizan las elecciones de Senado, para el cual se postulan como candidatos dos indígenas del departamento del Cauca: Anatolio Quirá Guauña, indígena kokonuco del municipio de Puracé, por la Alianza Social Independiente (ASI), y Floro Alberto Tunubalá, indígena misak, por las Autoridades Indígenas de Colombia (AICO); estos dos candidatos fueron elegidos para ser senadores en representación de los pueblos indígenas del Cauca.

Para las elecciones de la Cámara de Representantes se lanzaron por el departamento del Cauca Jesús Enrique Piñacué Achicué, indígena nasa del municipio de Inzá, por la ASI, y Luis Hernando Ramos Campo, por las AICO; sin embargo, estos dos candidatos no fueron elegidos. Laurent (2005), menciona que en este año se realizan las elecciones para gobernación, y como candidato se postuló a
Luis Guillermo Ramos Domínguez en representación de las AICO, pero sus votos fueron insuficientes para ser el gobernador del Cauca.

En 1992 se realizan elecciones para las alcaldías, asambleas departamentales y consejos municipales, pero las organizaciones indígenas solo postularon candidatos para alcaldías; en total fueron ocho candidatos indígenas en diferentes municipios, según la oficina del archivo de la Registraduría Departamental Cauca y la entrevista que se le hizo a Antonio Palechor Arévalo, indígena yanakona y comunicador del CRIC; los candidatos fueron: María Yalanda Campo en el municipio de Caldono, Abelardo Guegia Perdomo en el municipio de Páez, Evelio Correa Calambas en el municipio de Silvia, Manuel Dolores Chicangana Hormiga en el municipio de Sotará, José Venancio Dagua Mesa en el municipio de Toribío, Artenio Sánchez en el municipio de Totoró y finalmente Silvio Villegas en el municipio de Morales y Gilberto Muñoz Coronado también en el municipio de Toribío, quienes no eran indígenas propiamente pero hacían parte del movimiento y lo apoyaban fuertemente. Según Antonio Palechor, siete de ellos fueron elegidos para alcaldías.

En 1994, se realizaron las elecciones para gobernación, pero en esta ocasión no se lanzaron candidatos indígenas. No obstante, para las elecciones del Senado de este año los candidatos fueron Lorenzo Muelas Hurtado y Floro Alberto Tunubalá por las AICO y Jesús Enrique Piñacué Achicué por la ASI; los tres fueron elegidos. Para las elecciones de la Cámara de Representantes el candidato fue Anatolio Quirá Guauña por la ASI, pero no fue elegido. Para el año 1998 se lanzó nuevamente Jesús Piñacué por la ASI para el Senado y Anatolio Quirá también por la ASI para Cámara de Representantes. De acuerdo a lo anterior, el promedio de los candidatos indígenas del Cauca en los años 1991, 1992, 1994 y 1998 fue de cinco candidatos. Por otra parte, resulta importante hacer énfasis en el perfil académico de los candidatos a fin de definir sus niveles educativos y cómo estos generan cierta influencia en su vida política y se convierten en un aporte determinante para las organizaciones (figura 3).

El total de los candidatos que se graduaron del bachillerato en 1991 corresponde al 41,6 7\%; en 1992 al $25 \%$; en 1994 también el 25\% y en 1998 el 8,33 $\%$. De acuerdo con los datos, se observa que el nivel académico de los candidatos se va disminuyendo, pero se debe a que en el transcurso de estos años 
Figura 3. Promedio de candidatos graduados del bachillerato y universidad.

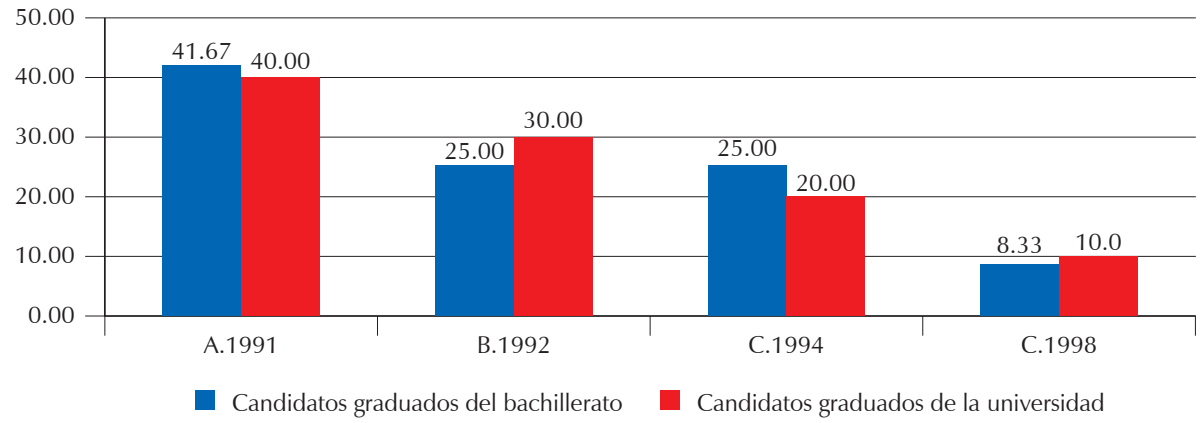

Fuente: Elaboración propia, a partir de información de las organizaciones de los movimientos indígenas en Colombia.

la mayoría de los candidatos son los mismos, por lo tanto en la gráfica se muestra que en 1991 hay más graduados, es decir, que para cada año solo se cuenta el nivel académico de los nuevos candidatos que se van lanzando en cada elección.

Finalmente, el promedio de los candidatos graduados de la universidad del $40 \%$ en 1991; del $30 \%$ en 1992; del 20 \% en 1994; y del 10 \% en 1998. Esta gráfica también muestra una disminución en el porcentaje de los graduados de la universidad, pero se debe a las mismas razones explicadas para los porcentajes de graduados de educación.

En concordancia con lo anterior, se evidencia que para la consolidación del movimiento indígena en el departamento del Cauca se tuvo que dar un proceso de organización, lucha y resistencia muy ardua por parte de todos los pueblos indígenas del Cauca, es decir, que la representación en el Congreso y en las alcaldías se gracias a las organizaciones que ellos mismos crearon, entre ellas las más antiguas e importantes está el CRIC, que desde 1971 viene impulsando el proceso político-organizativo del movimiento indígena no solo del Cauca sino también de toda Colombia.

\section{Nivel de votación que las comunidades indígenas tuvieron en las elecciones en el Departamento de Cauca entre 1991, 1992, 1994 y 1998}

Las comunidades indígenas del departamento Cauca se han venido consolidando con el pasar de los años en diferentes aspectos de la vida social: uno de ellos y quizá el más importante es la participación política y los procesos de vinculación en escenarios donde se tenga incidencia en las decisiones del país.

Uno de los alcances más importantes de las comunidades indígenas quedó consagrado en la Constitución, en su artículo 171, donde prevén dos escaños para "dos senadores elegidos por circunscripción nacional especial por comunidades indígenas". Quienes aspiran a ser elegidos en las circunscripciones indígenas deben “... haber ejercido un cargo de autoridad tradicional en su respectiva comunidad o haber sido líderes de una organización indígena, calidad que se acredita mediante certificado de la respectiva organización" (Laurent, 2015, p. 173.).

En los procesos de participación política, el elemento más importante o que se convierte en un símbolo de la democracia es el voto, el cual es el cumplimiento de un derecho así como el mecanismo mediante el cual los ciudadanos le dan la oportunidad a un candidato para que sea su representante. El voto es la medida de participación política más común en las democracias liberales, de esta manera es como "las democracias deben proporcionar a todos los ciudadanos oportunidades adecuadas e iguales para expresar sus preferencias y para incidir en las agendas decisionales de los gobiernos" (Dahl, 1989, p. 113).

Al respecto, Cuervo (2016) señala que el voto nos permite medir los niveles de participación, ya que responde a unas normas creadas de acuerdo a las necesidades de una sociedad determinada y no a un conjunto de normas estáticas. En el caso colombiano en la medida en que la democracia ha ido avanzando, ha obligado a evolucionar todo el 
sistema político de acuerdo al contexto histórico, económico y demográfico de la nación.

\section{Población votante}

Para las comunidades indígenas es de suma importancia el ejercer su derecho al voto, ya que lo ven como la forma en que las comunidades ceden sus voluntades a unos líderes que "atenderán la representación de los intereses de la población indígena del país" (Laurent, 1997b, p. 15, ya que estos individuos han tenido algún cargo o reconocimiento en su comunidad; así el líder se despoja de su autoridad tradicional para tener una inmersión en la arena política nacional, ya que este es uno de los requerimiento que se consagran en el artículo 171 de la Carta Constitucional. Como se indicó, en este artículo se determina que los representantes de las comunidades indígenas que aspiren a integrar el Senado de la República, deberán haber ejercido un cargo de autoridad tradicional en su respectiva comunidad o haber sido líder de una organización indígena, pues el hecho de haber ejercido un cargo en su comunidad les otorga legitimidad para así contar con una aceptación por parte de las comunidades para su representación.

Debemos reconocer además que los candidatos indígenas no solo obtienen votación de sus comunidades, sino que también cuentan con el apoyo significativo de otros grupos minoritarios y el resto de la sociedad; en este sentido, cuando se cumple con el deber tienen los ciudadanos de votar, se están encaminando o canalizando las fuerzas para la construcción de un Cauca incluyente, donde la apropiación de cada uno de los derechos y deberes que tienen las comunidades indígenas son la base de organización y autonomía para su desarrollo en el campo político.

De esta manera, es por medio del voto que se proyectan al resto de la población los ideales y metas que motivan la participación política de las comunidades, en concordancia con lo consagrado en el artículo 103 de la Constitución, donde se exponen los mecanismos de participación del pueblo, en el ejercicio de su soberanía, reflejada en acciones como el voto, el plebiscito, el referendo, la consulta popular, el cabildo abierto, la iniciativa legislativa y la revocatoria del mandato. En estos escenarios, la ley toma acciones para su reglamentación, de modo que también el Estado se convierte en un actor que contribuye a los procesos de organización, promoción y capacitación de las asociaciones de índole profesional, cívica, sindical, comunitaria y juvenil, sin que esto implique la pérdida de la autonomía con el objeto de que se constituyan mecanismos democráticos de representación.

En el departamento del Cauca, las elecciones del año 1991 para la Asamblea Nacional Constituyente, Senado, Cámara de Representantes y Gobernación, de 1992 para alcaldías, de 1994 para Cámara de Representantes, Senado y Gobernación, y de 1998 para Senado y Cámara de Representantes, nos arrojan los siguientes resultados (figura 4).

La población sufragante tiene un comportamiento ascendente, con una excepción en el año 1992, pero es necesario aclarar que en este año solo se

Figura 4. Promedio de la población sufragante

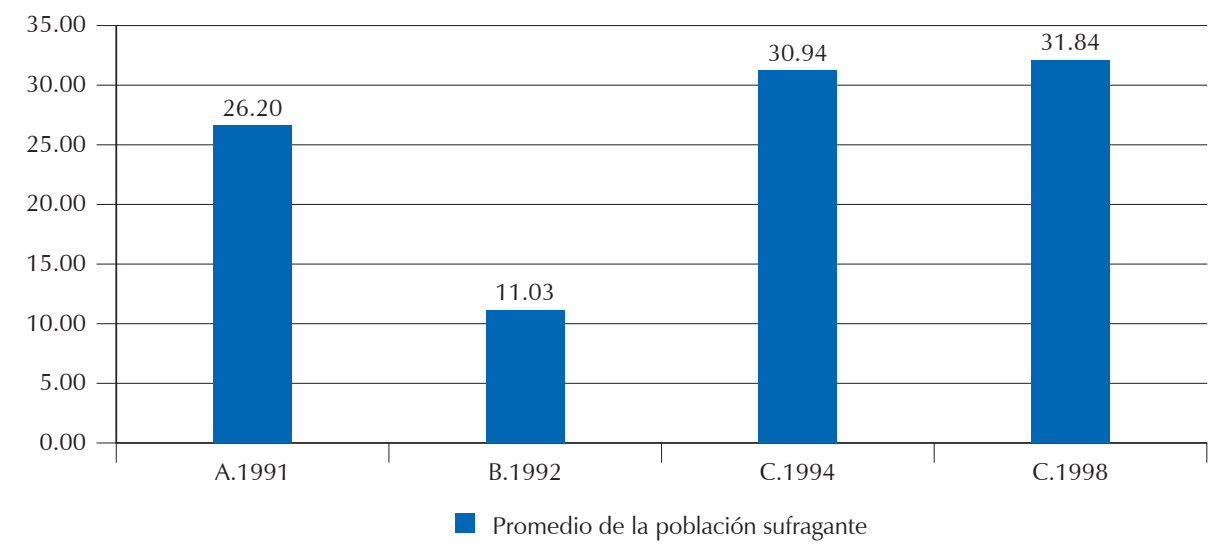

Fuente: Elaboración propia, a partir de la información hallada en el Archivo de la Registraduría Departamental del Cauca. 
está tomando en cuenta la elección de alcaldías municipales, que corresponde al 11,03 \%, lo que permite tener vislumbrar varios componentes, entre los cuales está el impacto de las elecciones legislativas, ya que se ve más movilización de población en las urnas; esto se debe a unos factores culturales, pues para muchos es más importante salir a votar para la elección de cargos importantes y que tienen repercusiones a nivel nacional.

El año 1991 es en el que hay mayor número de elecciones (Asamblea Nacional Constituyente, Senado, Cámara de Representantes y Gobernación), lo que deja en evidencia el hecho de que en la Asamblea Nacional Constituyente es la apertura a unos nuevos procesos políticos para las comunidades indígenas en Colombia, ya que por medio de estos procesos se deja ver cómo la población sufragante va en aumento, fenómeno que se ve claramente como en el último año, donde la participación votante es del 31,84 \%; así, con relación al primer año, es muy notable el avance que se da en la participación en procesos democráticos en el Cauca.

\section{Apoyo a las comunidades indígenas}

El apoyo a las comunidades indígenas se enfrenta a una multiplicidad de opciones a la hora de elegir; se deja ver una división en sectores opuestos a los partidos tradicionales, que limitan la votación a favor de los indígenas, ya que se catalogan sus campañas y gobierno con connotaciones de protesta; sin embargo, esto mismo motiva a varios grupos minoritarios a unirse a ellos y a apoyarlos por medio del voto. (Laurent, 1997a), lo cual tiene un gran significado para los representantes en sus comunidades, ya que estos procesos políticos se convierten en los portadores de su voz y necesidades.

Lorenzo Muelas considera de gran importancia el que apoyo brindado no solo por la comunidad indígena si no sino también por el resto del pueblo colombiano, no solo sea recordado como un dato histórico, sino que se convierta en un referente para crear conciencia de la importancia del respeto por el otro y de "la imperiosa obligación que todos tenemos de defender nuestros derechos y de respetar los de los demás" Banco de la Republica, 2017, p. 2).

Por ello, en varias asambleas los indígenas hacían comentarios como "nosotros necesitamos un representante que hable por nosotros, que defienda nuestros intereses y no debe desalentarnos el que no tengamos un doctor indígena" (Lame, 1916, p. 52). Estas palabras reflejan el discurso que por décadas llevaban manejando, y evidencian la importancia de la unidad para sacar un mayor número de votos en las urnas;.., estos se convierten en un medidor para saber la favorabilidad y aceptación de los líderes políticos indígenas en la nación., En el siguiente mapa se reflejan los porcentajes de participación por departamento a favor de los partidos políticos ONIC y AICO para las elecciones de Senado en el año 1991 (figura 5).

El nivel de votación es proporcional a la población apta para votar; los departamentos en donde se evidencia una gran concentración de votantes a favor del candidato indígena Gabriel Muyuy, en representación de la ONIC, son Cundinamarca, Córdoba, Antioquia, y en el sur del país los departamentos de Amazonas, Putumayo, Nariño, Cauca y Valle del Cauca; , este alto nivel de favorabilidad se debe a la alta presencia de comunidades indígenas en esta zona del país.; En el caso de las AICO, con el candidato Floro Tunubalá, que se lanzó en representación de las comunidades indígenas del Cauca, es evidente que el mayor número de votantes se concentra en la zona sur occidental del país, en los departamentos de Cauca, Valle del Cauca y Nariño, con la misma relevancia que tuvo la ONIC en Bogotá y Antioquia.

Tras las reformas a la Constitución se potenciaron el reconocimiento de las comunidades indígenas y la autonomía que se les cedió, para que pudieran vincularse a los procesos políticos; no se trata tan solo del reconocimiento por parte del Estado, sino que también hay un reconocimiento por el resto de la población, lo que lleva a que el apoyo a los candidatos indígenas no solo sea de indígenas, sino que se comienzan a vincular otros actores, generando un ambiente de inclusión e igualdad. Sin embargo, una de las reformas que generó inconformidad fue del modo o condiciones de elección que aplicaban específicamente para los representantes indígenas:

Durante los primeros quince años de la Constitución de 1991, los candidatos elegidos eran los que alcanzaban la mayor cantidad de votos para acceder a las curules aseguradas por dichas circunscripciones. Más allá de las críticas que podían despertar en cuanto a la validez de su acción. (Laurent, 2015, p. 176)

Las comunidades indígenas habían generado un buen grado de confianza en la población, lo que 
Figura 5. Votos Senado ONIC 1991
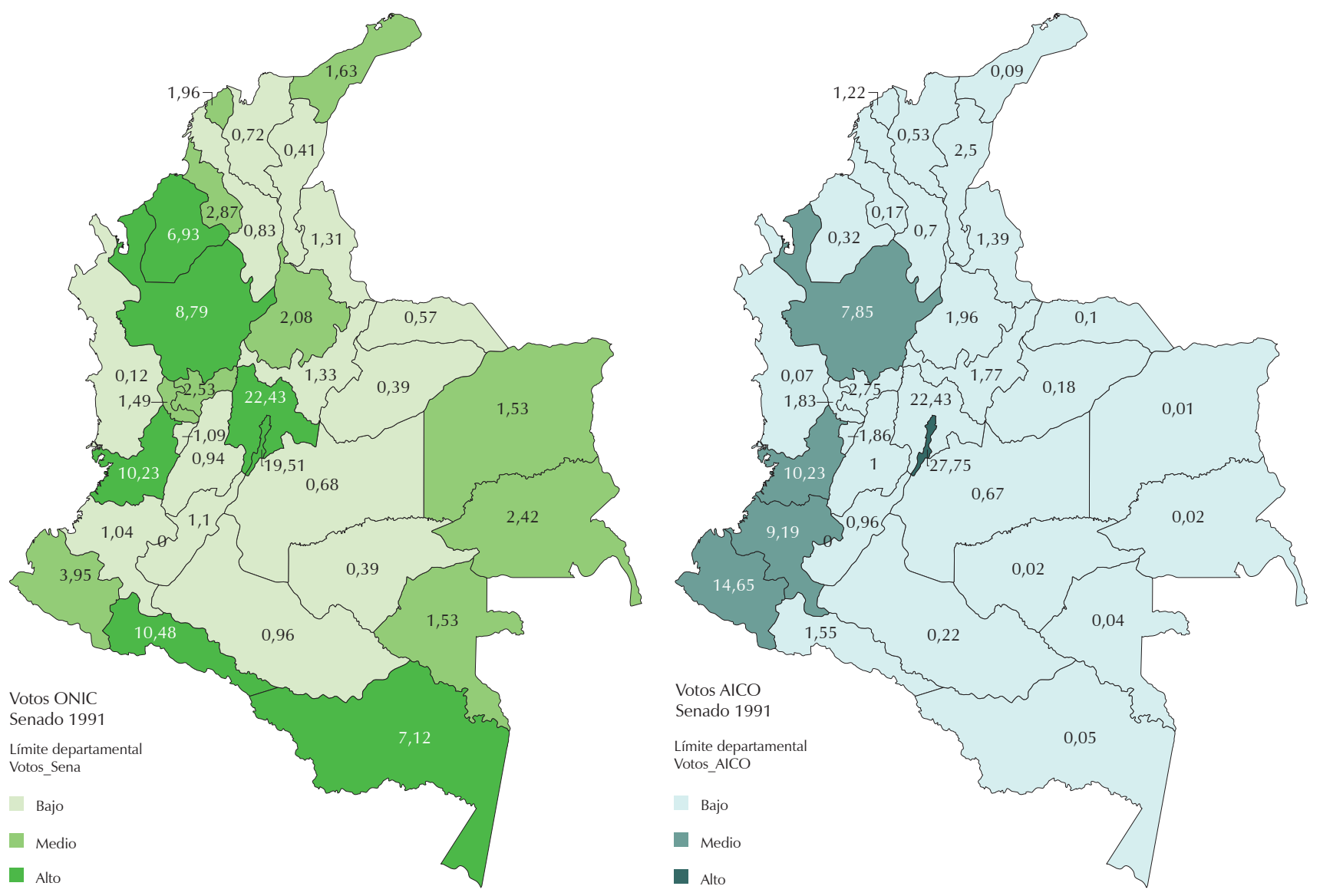

Fuente: Registraduria General de la Nación.

se vio reflejado en los niveles de favorabilidad de estos. En las elecciones del año 1991 los partidos políticos existentes eran AICO y ASI, en 1992 no se tiene información clara de los candidatos que se lanzaron a las alcaldías municipales, por lo cual no se tiene definido el partido político al cual pertenecían, en el año 1994 tienen representantes los partidos políticos AICO y ASI, y en el año 1998 los representantes del partido político ASI logran mover un buen capital electoral en lo que respecta a cada una de las elecciones (figura 6).

La favorabilidad de votación que se tiene para los candidatos indígenas del Cauca va en aumento; podemos ver cómo en cierta medida es ascendente, llevando a que su favorabilidad en el año 1991 sea del 27,19 \% y en el año de 1998 del 35,66 \%, lo que refleja un aumento notable en el recorrido. Esto se debe a que después de la Constituyente los candidatos indígenas del Cauca, comenzaron a tener cierto reconocimiento:

Tras la década de la entrada de Lorenzo Muelas y Francisco Rojas Birry en la asamblea constituyente de 1991, respectivamente en nombre del movimiento de Autoridades Indígenas de Colombia (AICO) y de la Organización Nacional Indígena de Colombia (ONIC), la década de los noventa dio paso a nuevas fuerzas políticas que se conformaron en aras de representar electoralmente a los pueblos indígenas. (Laurent, 2015, p. 170)

En la medida en que varios candidatos son elegidos, y el resto de la población los comienza a reconocer, estos adquieren cierto favoritismo y posicionamiento político entre los electores, lo que lleva a que el nivel de votación a favor de los candidatos indígenas vaya en aumento en los años posteriores a la Constituye. 
Figura 6. Promedio de votación a favor de los candidatos indígenas

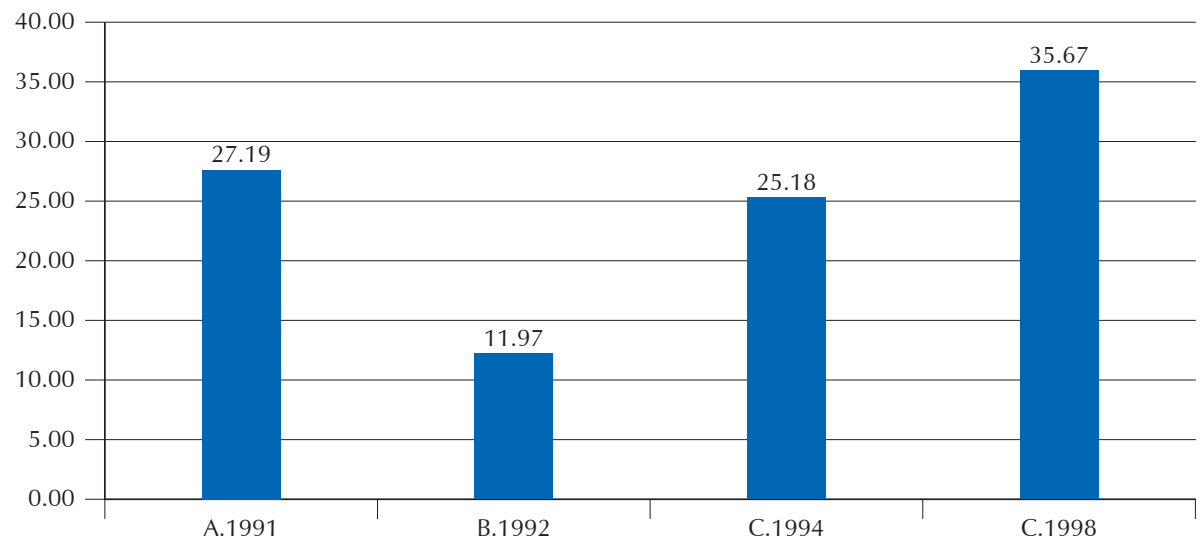

Fuente: Elaboración propia, a partir de la información hallada en el Archivo de la Registraduría Departamental del Cauca.

En su proceso de evolución en la participación política, de las comunidades indígenas han mantenido muy presentes varios elementos concernientes a sus cultura, uno de ellos es la relación de los rituales y la política, que Laurent (2005) considera como una actividad legitimadora hacia los candidatos, pues su ejercicio de gobierno adquiere un carácter sagrado.

Las comunidades se han agrupado y apoyan a sus candidatos, ya que para ellos la representación política garantiza la defensa de sus territorios, que es lo que les ha permitido organizarse e independizarse políticamente del resto de la sociedad, así como establecer vínculos y alianzas con otros grupos minoritarios, lo que llevó a que se convirtieran en una organización alternativa política para sectores que no son exclusivamente indígenas.

\section{Participación política de los} candidatos indígenas con respecto a los no indígenas y la incidencia de La Guajira en elecciones del Senado en los años 1991, 1994 y 1998

Colombia es un territorio pluriétnico en el que habitan aproximadamente ochenta grupos étnicos, estos grupos minoritarios que poseen un largo proceso organizativo y que han estado tradicionalmente en la arena política de nuestro país, principalmente las comunidades del departamento del Cauca, pues, como es sabido, el Cauca cuenta con una de las poblaciones de indígenas cuantitativamente más grandes de Colombia (Laurent, 1997b). Así como el Cauca, el departamento de La Guajira se ha caracterizado por los procesos de resistencia ante la violencia política y simbólica.

Otra de las constantes es que a los partidos políticos indígenas se les ha relacionado con partidos de izquierda, por la forma de su discurso y por sus procesos de lucha; esto ha llevado a que en muchas ocasiones se les consideren partidos que tienen nexos con grupos armados al margen de la ley.

Las alianzas que se han desarrollado entre partidos políticos de corte indígena definen parte de su lucha pues tienen como eje recuperar la autoridad y autonomía, elementos fundamentales para la reconquista del poder local en la que debe mediar la captación de recursos que permitan la ejecución de proyectos a favor de sus comunidades.

\section{Candidatos indígenas}

Con la Constitución de 1991, las comunidades indígenas tuvieron la oportunidad de empezar una nueva etapa en su gran movimiento, pues la participación por parte de los pueblos indígenas del Cauca en las elecciones de 1991, 1992, 1994 y 1998 fue la primera vez este movimiento tuvo que los pueblos indígenas tuvieron representación en diferentes cargos y espacios políticos de Colombia. Sin embargo, la participación de estos siempre ha sido mínima y restringida en comparación con la participación que tienen los demás partidos políticos, o para ser más puntuales, los partidos políticos tradicionales. 
El departamento del Cauca se encuentra en sur occidente del país, y La Guajira en la zona norte, en el Caribe, y tienen diferencias en la forma como se estructuran y referencian geopolíticamente; por esta razón, la participación política en cada uno de los departamentos está condicionada por unos lineamientos que cada movimiento u organización determinan en un plan de vida; sin embargo, revisando varios de estos lineamiento encontramos que concuerdan en un aspecto en específico: el reconocimiento cultural y político que se quiere tener en la nación.

Los candidatos indígenas han tenido que lidiar con varios factores que limitan su proceso de participación, entre los cuales se encuentran el ataque de grupos al margen de la ley., Es pertinente traer a colación el caso del departamento del Cauca, que ha sufrido la presencia de actores armados quienes "desde hace varios años han obligado a las comunidades indígenas a su presencia a su lado y que en los últimos tiempos pretendieron interferir directamente en el ejercicio político de los líderes del movimiento indígena" (Laurent, 1997b, p. 366).

Este tipo de injerencias limitan el desarrollo normal de las elecciones, pues en cierta medida hay influencia de estos grupos armados ilegales en la toma de decisiones, donde y en muchas ocasiones la población se siente atemorizada para salir a las urnas a ejercer su derecho al voto.

En las elecciones al Senado, el nivel de votación se puede ver a continuacion, donde se toman en cuenta los años 1991, que cuenta con el 27,.27\% de votación, el mismo porcentaje del año 1998; eleciones en las que los candidatos indígenas obtienen mayor votación son las de 1994, siendo reflejo del impulso que da la Constitución de 1991, que motivó un proceso de preparación y concientización de los movimientos y organizaciones, en el que desarrollan una andamiaje para obtener mejores resultados en 1994; en el año 1992 no se realizan elecciones de Senado, por lo cual el porcentaje es igual a cero (figura 7).

Los candidatos tienen una favorabilidad positiva en diferentes escenarios, ya que se establecen nuevas alianzas con grupos minoritarios que ayudan a establecer un reconocimiento favorable en la sociedad; sin embargo, los niveles votación desienden, debido en gran parte al incremento de la presencia y acciones de los grupos armados. De acuerdo con el Observatorio del Programa Presidencial de Derechos Humanos y DIH (2008), en los meses previos a la realización de comicios electorales del 26 de octubre de 1997, las FARC presionaron la renuncia de candidatos a los concejos y alcaldías en el $10 \%$ de los municipios del país. El sabotaje a las elecciones se produjo en localidades donde la guerrilla pretendía mostrar su influencia a través de la intimidación.

El Observatorio del Programa Presidencial de Derechos Humanos y DIH (2008) a explica que, al presionar la renuncia de los candidatos,: la subversión buscó impedir que avanzaran las estructuras de las autodefensas y que ejercieran dominio sobre los gobiernos locales, en las zonas donde estas estructuras se estaban fortaleciendo mediante el recurso a la violencia, en respuesta al vacío institucional antes mencionado, situación que favoreció el recurso a mecanismos de justicia privada por parte de la población civil (figura 8).

Pese a las diferentes circunstancias adversas, se logra que las curules tengan un buen avance, que es proporcional a la fuerza que toman las organizaciones en el país.

Figura 7. Promedio de votos obtenidos: Candidatos indígenas

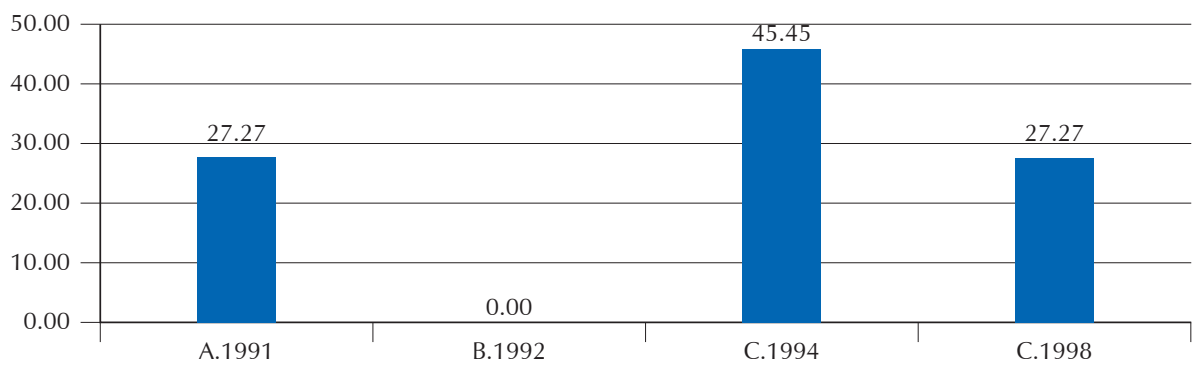

Fuente: Elaboración propia, a partir de la información hallada en el Archivo de la Registraduría Departamental del Cauca. 
Figura 8. Curules obtenidas por candidatos indígenas

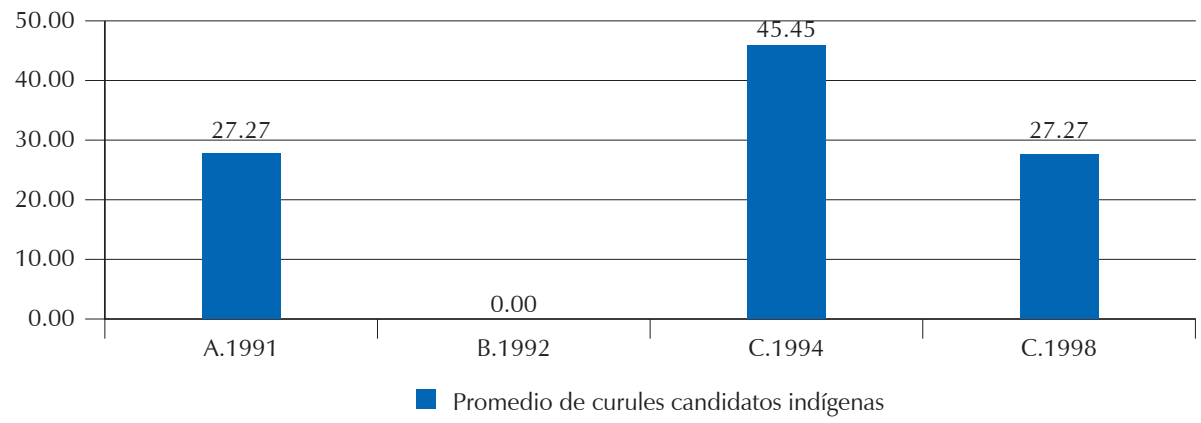

Fuente: Elaboración propia, a partir de la información hallada en el Archivo de la Registraduría Departamental del Cauca.

En el año 1994, los candidatos indígenas obtienen el $45,45 \%$ de las curules de los cuatro periodos de elecciones, siendo este promedio equivalente a cinco (5) curules y obteniendo el mismo número los años 1991 y 1998, con un 27,27 \%, con tres (3) curules para cada año.

La particiapcion electoral de los indígenas en general es buena, donde según los niveles de participación. Duque, (2014) menciona que la participación de las comunidades es relativamente exitosa y que con ella se logran escaños en el Congreso de la República, en las asambleas, concejos, alcaldías y gobernaciones; de igual manera, han tenido un desarrollo protagónico en movilizaciones de minorías indígenas y no indígenas, y de esta manera logran hacerse visibles en escenarios del poder político.

\section{Candidatos no indígenas}

Los resultados que presentan los candidatos no indígenas son relativamente mayores, debido a diferentes factores entre los cuales se destaca la trayectoria de los partidos tradicionales Conservador y Liberal, que tienen sus orígenes en el proceso de independencia de Colombia con el general Santander y El Libertador Simón Bolívar. Después, los procesos de organización de grupos minoritarios llevaron a la aparición de nuevos partidos y, de esta manera, se inicia un proceso de división entre las fuerzas partidistas donde se comienza a hablar de derecha e izquierda, lo que el escenario político se polariza, y tanto candidatos como electores son conscientes de que deben optar por uno de los dos lados; esta es la forma como el sistema genera las condiciones para que existan dos polos.
Sin embargo, se han presentado diferencias y fragmentaciones entre las organizaciones indígenas, lo cual ha llevado a que se agrupen según sus intereses; así como lo mencioné anteriormente, las diferentes organizaciones han tenido diferencias de tipo económico, ideológico económico, ideológico y cultural; una de ellas gira en torno al principio mencionado de "recuperar los espacios vitales del medio ambiente, a partir de la idea según la cual uno es parte y no dueño de la tierra" (Laurent, 2005, p. 5).

La población de La Guajira es evidentemente la representación de la periferia, dada su ubación geográfica y a que sus comunidades han sido por años, quedando así aisladas del Estado y presentando unas brechas de desigualdad proporcionalmente marcadas que han llevado a que "en los territorios de La Guajira los indígenas están siendo desalojados progresivamente y los nativos se han visto forzados a emigrar más y más hacia el norte, donde las tierras son más estériles." (Sánchez y Molina, 2010, p. 23).

Es un caso muy particular el del departamento de La Gaujira, pues siendo un territorio con una población indígena alta, llama la atención la poca votación a favor de los candidatos indígenas; al respecto, Laurent (1997a) menciona que la poca participación de los candidatos de las organizaciones "indígenas" en las últimas elecciones podría traducir cierta indiferencia, o una perspectiva diferente a la acción de participar.

En las elecciones del Senado, la votación que obtienen los partidos tradicionales con respecto a los partidos de comunidades indígenas es 
Figura 9. Votación por partidos senado 1991

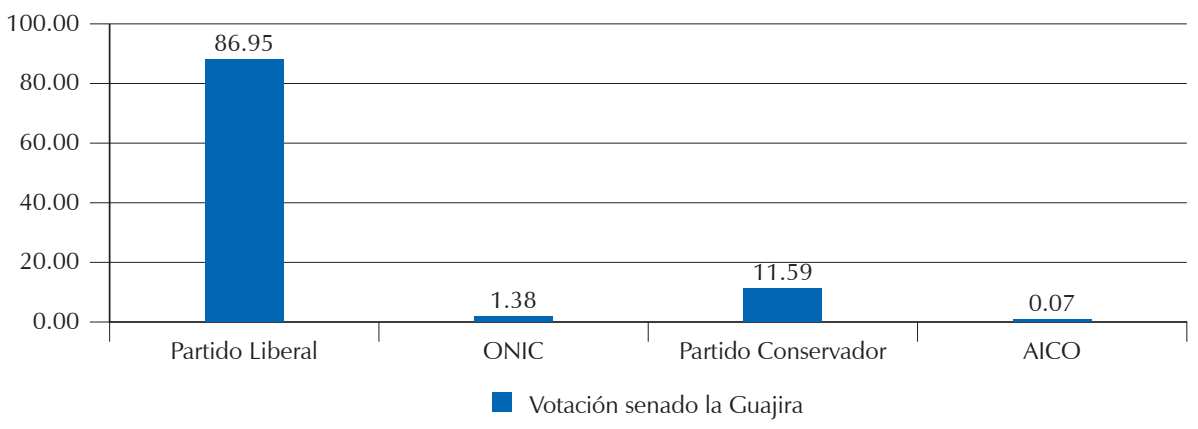

Fuente: Elaboración propia, a partir de la información hallada en el Archivo de la Registraduría Departamental del Cauca.

evidentemente mayor, ya que ninguno de los dos los partidos indígenas no alcanzan ninguno de los dos alcanza el $2 \%$, en tanto que el Partido Liberal obtiene el 86,95\%, siguiéndole el Partido Conservador por encima del $11 \%$; esto puede tener varios factores de incidencia, como el andamiaje de los partidos tradiconales que los ha llevado a generar un apoyo más notable por parte de la población., Pero debemos tener en claro que estamos hablando de un territorio indígena donde se supone que al haber la opcion de elegir entre partidos tradicionales y partidos indígenas ellos deberían votar por los partidos que representen sus intereses.

Otro de los casos sucedió en La Guajira en 1992; se cree que la falta de apoyo a los partidos políticos indígenas está ligada a una pérdida de respeto por las autoridades tradicionales, que se vio reflejada en las elecciones. Así, en para las comunidades wayuu los resultados de los candidatos indígenas han quedado muy lejos de las expectativas después de unos esfuerzos de participación prometedores en las elecciones municipales del 92" (Laurent, 1997a, p.).

\section{CONCLUSIONES}

Los avances en tema normativo se presentan como garantes para el desarrollo de escenarios de participación política de los indígenas, si bien no han estado acompañados de un monitoreo o seguimiento que permita hacer ajustes que ayuden a lograr el objetivo con el que se diseñaron. No hay que negar que el trabajo de las comunidades indígenas es sumamente importante y ha cobrado relevancia, pero es necesario que sea una construcción bilateral, en donde se establezca la ley y de igual manera las condiciones para su cumplimiento.

Los esfuerzos de las comunidades indígenas se comienzan a ver reflejados en que sus partidos comienzan a ser referentes en sus procesos de resistencia, lo que lleva a que diferentes personas se comiencen a alinear con sus ideales y a apoyarlos en sus procesos de participación; de esta forma, se establecen alianzas en busca de cooperación económica, política y social, para la conservación de territorios y para prevenir la explotación de los recursos naturales del país.

El proceso de elección de candidatos indígenas ha estado guiado por un factor identitario; se pretende que los líderes promuevan una reivindicación entre el Estado y las comunidades, y que se les asigne lo que les corresponde como ciudadanos y grupos étnicos. Este proceso, sin embargo, no se da de la noche a la mañana, y debe estar condicionando por cambios de factores culturales de largo alcance en un trabajo continuo y constante que permita hacer política desde la diversidad. 


\section{REFERENCIAS}

Banco de la República. (2017). Lorenzo Muelas. http://enciclopedia.banrepcultural.org/index. php/Lorenzo_Muelas

Castoriadis, C. (1997). El imaginario social instituyente. Zona Erógena, 35.

CRIC. (2007). Plan de vida de los pueblos indígena del Cauca. Cauca.

Cuervo, J. J. (2016). El derecho humano al voto. México D. F.: Comisión Nacional de los Derechos Humanos-CNDH.

Dahl, R. (1989). Democracy and its critics.

Duque, J. (2014). Las organizaciones políticas étnicas en Colombia. Los indígenas y las elecciones 1990-2006. Iberoamericana, 7-30.

Lame, M. Q. (1916, 2 de junio). Nota periodística Manuel Quintin Lame.

Laurent, V. (1997). Pueblos indígenas y espacios políticos en Colombia: tendencias nacionales, diferencias regionales. Quito.

Laurent, V. (1997a, 17 de julio). Noticias de antropología y arqueología.
Laurent, V. (2015). Elecciones 2014 y movilizacion politica indígena: apuestas, ajuste y $\dot{i}(r e)$ conciliación? Bogota: Konrad Adenaur Stiftung.

Laurent, Virginie. (2005). Comunidades indígenas, espacios políticos y movilización electoral en Colombia, 1990-1998. Motivaciones, campos de acción e impactos. Bogotá: Instituto Francés de Estudios Andinos.

Ministerio del Interior. (2016). A/CO-Autoridades Indígenas de Colombia. https://www.mininterior.gov.co/content/aico

Observatorio del Programa Presidencial de Derechos Humanos y DIH. (2008). Impacto de la política de seguridad democrática. Sobre la confrontacion armada, el narcotráfico. Bogotá: Observatorio del Programa Presidencial de Derechos Humanos y DIH.

Sánchez Gutiérrez, E. y Molina Echeverri, H. (2010). Documentos para la historia del movimiento indígena colombiano contemporáneo. Bogotá: Ministerio de Cultura. 